\title{
Über das Schildpatt von Chelone imbricata.
}

Von

Hans Buchtala.

(Aus dem Institute für medizinische Chemie der Universität Graz.)

(Der Redaktion zugegangen am 28. Juli 1911.)

In früheren Arbeiten konnte gezeigt werden, daß bei Horngebilden nicht nur verschiedener Tierarten, sondern sogar ein und desselben Tieres beträchtliche Unterschiede in bezug auf einen schwefelhaltigen Baustein, das Cystin, obwalten. Da derartige Unterschiede bezüglich der anderen Bausteine der wenigen bisher untersuchten Keratine nicht zutage getreten sind, habe ich auf Anregung meines hochgeschätzten Lehrers und Vorstandes, des Herrn Hofrates K. B. Hofmann, die Untersuchung epidermoidaler Horngebilde verschiedener Tiergattungen in Angriff genommen. Da über Hornsubstanzen von Reptilien noch keine Untersuchungen vorliegen, habe ich zunächst das leichter zugängliche Schildpatt von Chelone imbricata in Arbeit genommen. ${ }^{1}$ ) Das zur Untersuchung verwendete Schildpatt wurde zuerst mit einprozentiger Salzsäure, hierauf mit heißem Wasser gewaschen und endlich mit Ätheralkohol längere Zeit am Rückflußkühler ausgekocht.

Wasser- und Aschegehalt.

$0,7828 \mathrm{~g}$ verloren beim Trocknen bei $105^{\circ}$ im Vakuum 0,0739.g an Gewicht, entsprechend 9,44\% Wasser.

Beim Glühen hinterblieb eine weiße Asche im Gewichte von $0,0021 \mathrm{~g}$. Dieselbe zeigte sich im Wasser nur teilweise löslich und bestand aus Calcium, Spuren von Eisen, sowie Phosphorsäure und Schwefelsäure.

1) Das Material wurde uns von Herrn E. Rzib a, Besitzer des großen Geschäftes für Schildpattwaren in Wien, in freundlicher Weise zur Verfügung gestellt, wofür ihm auch an dieser Stelle der beste Dank gesagt sei. 
Verhalten gegen Alkali und heißes Wasser.

In zehnprozentiger Natronlauge löst sich eine Probe ziemlich leicht mit gelber Farbe auf, und beim Neutralisieren mit Säure fällt ein flockiger Niederschlag aus, der sich rasch zusammenballt. Die Lösung selbst gibt die Millon sche und die Biuretreaktion.

Drei Gramm von Schildpattspänen wurden mit $80 \mathrm{ccm}$ destillierten Wassers in einem Porzellantiegel drei Stunden lang im Autoklaven auf $120^{\circ}$ erhitzt. Die Masse schrumpfte zu einem sehr porösen Klumpen zusammen, der im Aussehen vollkommen einem Schwamme glich, und auch wie ein solcher nach dem Auspressen der Flüssigkeit Wasser wieder begierig aufsog. Die wässerige Lösung zeigte schon die Reaktion von Molisch; die Tryptophanreaktion nach Rhode fiel negativ aus. Die vereinigten wässerigen Lösungen hinterließen beim Verdampfen nur $0,07 \mathrm{~g}$ trockenen Rückstand.

\section{Stickstoffverteilung im Schildpatt.}

Zur Orientierung über das Verhältnis von Mono- zu Diaminosäuren im Schildpatt wurde die Verteilung des Stickstoffes nach der Methode von Hausmann ${ }^{1}$ ) beziehungsweise nach Gümbel²) untersucht.

Das untersuchte Schildpatt zeigte im lufttrockenen $\mathrm{Zu}$ stande einen Gehalt von 14,14\% Stickstoff.

$1,1221 \mathrm{~g}$ verbrauchten bei der Bestimmung nach $\mathrm{Kjel-}$ dahl $56,5 \mathrm{ccm} n / 5-\mathrm{HCl}=14,10 \% \mathrm{~N}$.

$0,8705 \mathrm{~g}$ verbrauchten bei der Bestimmung nach $\mathrm{Kjel-}$ dahl $44,1 \mathrm{ccm} n / 5-\mathrm{HCl}=14,18 \% \mathrm{~N}$.

Von diesem Präparate wurden $4 \mathrm{~g}$ in Arbeit genommen. Das Ammoniak des Filters samt Niederschlag verbrauchte 2,1 ccm n/10-Säure $=0,07 \%$ des Präparates oder 0,52\% des Gesamtstickstoffes.

Der in Lösung gebliebene Teil gab nach dem Abdestillieren der Salzsäure bei überschüssigem Zusatz von Magnesium-

1) Diese Zeitschrift, Bd. 27, S. 95, und Bd. 29, S. 136.

2) Hofmeisters Beiträge, Bd. 5, S. 297. 
oxyd so viel Ammoniak ab, daß dieses $12,3 \mathrm{ccm}$ n/10-Säure verbrauchte. Die Menge des abgespaltenen Ammoniakstickstoffes beträgt sonach $0,43 \%$ des Präparates oder 3,04\% des Gesamtstickstoffes.

Zum Zwecke der weiteren Bestimmungen wurde der Destillationsrückstand in Salzsäure gelöst und der Kolbeninhalt auf $500 \mathrm{ccm}$ mit Wasser aufgefüllt. Je $25 \mathrm{ccm}$ verbrauchten 19,6 bezw. $19,5 \mathrm{ccm} \mathrm{n} / 10$-Säure, entsprechend $13,72 \%$ des Präparates oder 97,03 des Gesamtstickstoffes.

Die Phosphorwolframsäureniederschläge verbrauchten bei je $100 \mathrm{ccm}$ des Filtrates $2,5 \mathrm{ccm} \mathrm{n} / 10$ Säure $=0,44 \%$ des Präp. oder 3,09 des Gesamtstickstoffes.

Die Filtrate verbrauchten übereinstimmend $19,6 \mathrm{ccm}$ n/5-Säure $=13,41 \%$ der Präp. oder 94,84\% des Ges. Stickst.

Übersichtstabelle.

\begin{tabular}{l|c|c}
\hline & \multicolumn{2}{|c}{ Prozente } \\
& der Probe des Schildpattes & des Gesamtstickstoffes \\
\hline Melaninstickstoff . . & 0,07 & 0,02 \\
Ammoniakstickstoff . & 0,43 & 3,04 \\
Monoaminostickstoff . & 13,41 & 94,84 \\
Diaminostickstoff . . & 0,44 & 3,09
\end{tabular}

Wie aus der Tabelle ersichtlich ist, treten die Diaminosäuren beim Schildpatt ganz in den Hintergrund; ich beschränkte mich daher bei der weiteren Untersuchung auf die Darstellung der Monoaminosäuren.

Hydrolyse des Schildpattes mittels Salzsäure.

Die Hydrolyse wurde zweimal mit je $350 \mathrm{~g}$ des lufttrockenen Präparates durchgeführt. Die erfreulicherweise fast ganz übereinstimmenden Resultate sind ein neuerlicher Beweis für die Vortrefflichkeit und Brauchbarkeit der Fischerschen Estermethode bei vergleichenden Untersuchungen von E:weißkörpern. Es sei gleich von vornherein mitgeteilt, daß bei der ersten Hydrolyse $102 \mathrm{~g}$ Glykokollesterchlorhydrat und $268 \mathrm{~g}$ eines Gemenges anderer Ester, bei der zweiten Hydrolyse $112 \mathrm{~g}$ 
Glykokollesterchlorhydrat und $243 \mathrm{~g}$ Ester erhalten wurden. Der Gehalt an einzelnen Aminosäuren schwankt bei beiden Proben nur unbeträchtlich; da die Trennung derselben jedoch bei der zweiten Hydrolyse glatter vonstatten ging, sollen, schon der Einheitlichkeit wegen, bloß die Resultate der letzteren dieser Arbeit zugrunde gelegt werden.

Die, wie früher erwähnt, gereinigte Substanz im Gewichte von $350 \mathrm{~g}$ wurde mit $1100 \mathrm{ccm}$ konzentrierter Salzsäure durch acht Stunden am Rückflußkühler gekocht. Als Rückstand hinterblieb eine schwammige Masse im Gewichte von 6,2 g. Die nach möglichstem Entfernen der Salzsäure zurückbleibende Masse wurde zweimal mit je $1 \mathrm{l}$ absoluten Alkohols verestert und die Esterlösung im Vakuum auf die Hälfte verringert. Nach 24 stündigem Stehen unter Kühlung war die Masse ganz erstarrt und wurde nach weiteren 24 Stunden abgesaugt. Die schwach gelbliche Krystallmasse von Glykokollesterchlorhydrat hatte nach dem Trocknen im Vakuum über Schwefelsäure und gebranntem Kalk ein Gewicht von $112 \mathrm{~g}$. Nach einmaligem Umkrystallisieren aus Alkohol und Entfärbung mit Tierkohle war der Körper fast rein weiß. Die feinen Nadeln schmolzen bei $144^{\circ}$.

0,2171 g Substanz verbrauchten 56,30 ccm einer Lösung von $\mathrm{AgNO}_{3}$, von der $1 \mathrm{ccm}$ einem $\mathrm{mg} \mathrm{Cl}$ entspricht.

Sonach: Gefunden $25,93 \% \mathrm{Cl}$.

Berechnet für $\mathrm{C}_{4} \mathrm{H}_{9} \mathrm{O}_{2} \mathrm{NHCl} 25,43 \% \mathrm{Cl}$.

Die vom Glykokollesterchlorhydrat getrennte Mutterlauge wurde im Vakuum möglichst eingeengt und die Ester durch Kalilauge und Kaliumcarbonat in Freiheit gesetzt. Die ätherischen Auszüge waren stark gelbbraun gefärbt und wurden mit Kaliumcarbonat und geglühtem Natriumsulfat getrocknet. Der breiige Rückstand wurde mit dem Salzsäurealkohol, der von früheren Veresterungen gesammelt war, übergossen, wodurch eine fast vollständige Scheidung der anorganischen Bestandteile erzielt wurde. Die von diesen abgesaugte Lösung wurde unter geringem Druck vollständig verdampft und der Rückstand abermals verestert und dieser Vorgang noch ein drittes Mal wiederholt. Es gelingt auf diese Weise, in verhältnismäßig kürzerer 
Zeit als nach den früheren Verfahren wiederholte Veresterungen durchzuführen. Schließlich wurden die Ester noch mit Chloroform extrahiert, eine Methode, die jüngst von Abderhalden angegeben wurde; es ging in diesem Versuche fast nur der Ester des Tyrosins über, wie ich mich durch nähere Untersuchung des Extraktes überzeugen konnte und wie auch $\mathrm{Ab}$ derhalden davon Erwähnung tut. ${ }^{1}$ )

Nach dem Abdestillieren des Äthers verblieb ein Estergemenge im Gewichte von $243 \mathrm{~g}$, das der fraktionierten Destillation bei einem Druck von $11 \mathrm{~mm}$ unterworfen ward. Dabei ergaben sich folgende Ausbeuten:

1. Fraktion bis $60^{\circ}$ (Temperatur des Wasserbades) $=60 \mathrm{~g}$

2. $\gg 80^{\circ}$

$3 . \gg 105^{\circ}$

$4 . \quad \gg 180^{\circ}$

5. Destillationsrückstand

$=14,3$ » $=66,0$ » $=15,0$ » $=87,0$ »

I. Fraktion (bis $60^{\circ}$ ).

Dieselbe bestand hauptsächlich aus Alkohol und Chloroform neben einem Rückstand von $2 \mathrm{~g}$, der sich nach dem Verseifen mit Wasser als Glykokoll erwies (F. 240 $)$.

\section{Fraktion $\left(60-80^{\circ}\right)$.}

Aus dieser Fraktion wurden nach 5 stündigem Kochen mit $100 \mathrm{ccm}$ Wasser $5,2 \mathrm{~g}$ einer rein weißen Substanz erhalten, welche bei $280^{\circ}$ schmolz und zum größten Teil aus Alanin bestand. Es konnten daraus durch fraktionierte Krystallisation $4,5 \mathrm{~g}$ Alanin gewonnen werden. Das Alanin zeigte einen Schmelzpunkt von $293^{\circ}$. Bei der Stickstoffbestimmung nach Dumas im Mikroverbrennungsapparate nach Pregl wurde folgendes Resultat erhalten: $12,60 \mathrm{mg}$ lieferten $1,98 \mathrm{ccm} \mathrm{N}$ bei $735 \mathrm{~mm} \mathrm{Hg}$ und $22,5^{\circ} \mathrm{C}$.

Die gefundene Stickstoffmenge beträgt $15,72 \%$ gegenüber der berechneten von $15,74 \%$ für $\mathrm{C}_{3} \mathrm{H}_{7} \mathrm{O}_{2} \mathrm{~N}$.

Der Rest dieser Fraktion im Gewichte von $0,7 \mathrm{~g}$ bestand aus Glykokoll.

1) Diese Zeitschrift, Bd. 71, S. 457. 


\section{Fraktion $\left(80-105^{\circ}\right.$.)}

Bei der Verarbeitung dieser Fraktion w urde die Erfahrung Pregls ${ }^{1}$ ) berücksichtigt, daß der Leucinester dem mit Wasser verdünnten Estergemenge entzogen werden kann, während die Ester der übrigen Aminosäuren in der wässerigen Lösung bleiben. Bei der weiteren Verarbeitung der Ätherlösung zeigte es sich jedoch, daß auch der Valinester leicht in den Äther überging. Der Destillationsrückstand der Ätherausschüttelungen wurde mit der sechsfachen Menge Wasser durch 10 stündiges Kochen verseift. Nach 12 stündigem Stehen bei Zimmertemperatur schied sich eine weiße Krystallmasse ab, die ausschließlich aus Leucin bestand und $8,5 \mathrm{~g}$ wog. Das Leucin schmolz bei $297^{\circ}$. Das daraus dargestellte Kupfersalz war zum Teil in Methylalkohol löslich, bestand also teilweise aus Isoleucinkupfer.

$0,1496 \mathrm{~g}$ Substanz lieferten 0,0364 $\mathrm{g} \mathrm{CaO}$.

Berechnet für $\left(\mathrm{C}_{6} \mathrm{H}_{12} \mathrm{NO}_{\mathbf{8}}\right)_{8} \mathrm{Cu}$ :

Gefunden:

$19,64 \%$ $19,43 \% \mathrm{Cu}$.

Der Rest des Leucins wurde mit absolutem Alkohol ausgekocht. Mit der verbleibenden Krystallmasse wurde eine Stickstoffbestimmung vorgenommen.

$14,75 \mathrm{mg}$ lieferten $1,62 \mathrm{~cm} \mathrm{~N} . p=733 \mathrm{~mm}, \mathrm{t}=22^{\circ} \mathrm{C}$. Berechnet für $\mathrm{C}_{6} \mathrm{H}_{13} \mathrm{NO}_{2}$ : Gefunden: $10,69 \% \mathrm{~N} \quad 10,96 \% \mathrm{~N}$.

Die Mutterlauge vom Leucin wurde bis zur Trockene verdampft, der Rückstand mit absolutem Alkohol ausgekocht und die alkoholischen Auszüge vereinigt. Der in Alkohol unlösliche Anteil wurde in Wasser gelöst und in zahlreiche Fraktionen zerlegt: er bestand der Hauptmenge nach, wie die Analyse beweist, aus Valin, von dem $17 \mathrm{~g}$ isoliert wurden. $\mathrm{t}=22^{\circ} \mathrm{C}$.

$9,15 \mathrm{mg}$ Substanz lieferten $1 ; 09 \mathrm{ccm} \mathrm{N} ; \mathrm{p}=739 \mathrm{~mm}$,

$$
\begin{array}{cc}
\text { Berechnet für } \mathrm{C}_{5} \mathrm{H}_{11} \mathrm{NO}_{2}: & \text { Gefunden: } \\
11,97 \% \mathrm{~N} & 11,97 \% \mathrm{~N} .
\end{array}
$$

1) Diese Zeitschrift, Bd. 52, S. 459.

Hoppe-Seyler's Zeitschrift f. physiol. Chemie. LXXIV. 


\section{Optische Bestimmung.}

$0,3322 \mathrm{~g}$ wurden in $20 \mathrm{ccm} 21,5 \%$ iger Salzsäure gelöst. Im Dezimeterrohr wurde der Winkel $\alpha=+0,32^{\circ}$ abgelesen.

$$
[\alpha]_{\mathrm{D}}^{20^{\circ}}=+19,26^{\circ}
$$

Das Valin schmolz im Kapillarrohr bei $135^{\circ}$ (unkorrigiert). Die letzten zwei Fraktionen im Gewichte von $1,4 \mathrm{~g}$ bestanden fast nur aus Alanin mit dem Schmelzpunkt $295^{\circ}$.

Aus der wässerigen Lösung der dritten Fraktion wurde nach dem Verseifen mit der zehnfachen Wassermenge eine Krystallmasse erhalten, die im wesentlichen nur aus Alanin bestand. Nach dem Auskochen mit absolutem Alkohol konnten daraus $3,7 \mathrm{~g}$ Alanin isoliert werden.

Die vereinigten alkoholischen Auszüge sollten zur Darstellung der $\alpha$-Pyrrolidincarbonsäure dienen. Es wurde dreimal im Vakuum der Alkohol abdestilliert und der Rückstand jedesmal in absolutem Alkohol aufgenommen. Schließlich verblieb nur eine Masse von sirupartiger Konsistenz, die sich in der Kälte leicht in Alkohol löste. Der Versuch, über das Kupfersalz einen krystallisierten analysierbaren Körper zu erhalten, mißlang; der schmierige Abdampfungsrückstand trotzte jedweden Krystallisierungsversuchen.

\section{Fraktion.}

Das Estergemenge dieser Fraktion wurde nach dem Verdünnen mit Wasser mit Äther ausgeschüttelt. Nach dem Abdestillieren des Äthers und Verseifen des Rückstandes mit konzentrierter Salzsäure wurden $3,66 \mathrm{~g}$ Phenylalaninchlorhydrat erhalten. Das Phenylalanin, das daraus durch überschüssigen Zusatz von Ammoniak erhalten wurde, schmolz unter Zersetzung bei $283^{\circ}$.

$11,40 \mathrm{mg}$ Substanz lieferten $1,01 \mathrm{ccm} \mathrm{N} ; \mathrm{p}=736 \mathrm{~mm}$, $\mathrm{t}=22^{\circ} \mathrm{C}$.

$$
\begin{array}{cl}
\text { Berechnet für } \mathrm{C}_{9} \mathrm{H}_{11} \mathrm{NO}_{2}: & \text { Gefunden: } \\
8,49 \% \mathrm{~N} & 8,87 \% \mathrm{~N} .
\end{array}
$$

Aus der wässerigen Lösung dieser Fraktion konnte weder Glutaminsäure noch Asparaginsäure mit Sicherheit isoliert werden. 


\section{Destillationsrückstand.}

Derselbe wurde mit überschüssigem Baryt und zugesetzter Tierkohle 8 Stunden lang erhitzt. Es gelang nicht, nach Entfernen des Baryts im Filtrate Glutaminsäure zu finden.

Bei einem eigens zu diesem Zwecke mit $50 \mathrm{~g}$ Schildpatt angestellten Versuch konnte trotz vollständigen Entfärbens des Hydrolysates mit Tierkohle und weiters noch durch Schütteln mit Kupferoxydul und Fällen mit Schwefelwasserstoff keine Glutaminsäure aufgefunden werden. Es scheint demnach dieser Baustein der Keratine dem Schildpatt zu fehlen.

$$
\text { Schwefel- und Cystingehalt. }
$$

Die Schwefelbestimmung wurde durch Schmelzen der Substanz mit Salpeter und Natriumhydroxyd in der bekannten Weise ausgeführt.

$$
\begin{array}{rl}
1,6898 \mathrm{~g} \text { Substanz gaben } 0,1931 & \mathrm{~g} \mathrm{BaSO}_{4}=1,57 \% \mathrm{~S} \\
2,1264,0.2208 * \mathrm{BaSO}_{4} & =1,43 \% \mathrm{~S} \\
\text { im Mittel } & =1,50 \% \mathrm{~S} .
\end{array}
$$

Für die Cystinbestimmung wurden $100 \mathrm{~g}$ Substanz hydrolysiert. Der Cystinniederschlag wurde mit Ammoniak in Lösung gebracht und die Lösung auf $500 \mathrm{ccm}$ ergänzt. Aus $10 \mathrm{ccm}$ dieser Lösung wurden nach der Schmelze 0,1884 $\mathrm{g} \mathrm{BaSO}$ erhalten. Die aus dem Schwefelgehalt des Cystinniederschlages berechnete Cystinmenge beträgt in bezug auf die getrocknete Substanz 5,19\%.

\section{Bestimmung des Tyrosins.}

$70 \mathrm{~g}$ Schildpatt, entsprechend 61,81 g Asche und wasserfreier Substanz, wurden mit $200 \mathrm{~g}$ konzentrierter Schwefelsäure und $400 \mathrm{ccm}$ Wasser 12 Stunden hindurch gekocht. Nach dem Entfernen der Schwefelsäure mit Baryt und wiederholtem Auskochen des Niederschlages wurden aus der wässerigen Lösung beim Einengen und Abkühlen 11,2 g Rohtyrosin erhalten. Dieses wurde durch Auskochen mit Eisessig und Umkrystallisieren aus heißem Wasser gereinigt. Es wurden so $8,4 \mathrm{~g}$ reines Tyrosin erhalten. 
220 Hans Buchtala, Über das Schildpatt von Chelone imbricata.

$\begin{array}{cc}7,37 \mathrm{mg} \text { lieferten } 0,57 \mathrm{~cm} \mathrm{~N} ; \mathrm{p}=734 \mathrm{~mm}, \mathrm{t}=23^{\circ} \mathrm{C} \text {. } \\ \begin{array}{c}\text { Berechnet für } \mathrm{C}_{9} \mathrm{H}_{11} \mathrm{NO}_{3}: \\ 7,74 \% \mathrm{~N}\end{array} & \text { Gefunden : } \\ & 7,73 \% \mathrm{~N} .\end{array}$

Übersicht der Resultate:

\begin{tabular}{lr} 
Glykokoll & $19,36 \%$ \\
Alanin & $2,95 \%$ \\
Valin & $5,23 \%$ \\
Leucin & $3,26 \%$ \\
Phenylalanin & $1,08 \%$ \\
Tyrosin & $13,59 \%$ \\
Cystin & $5,19 \%$ \\
\cline { 2 - 2 } & $50,66 \%$
\end{tabular}

Ein vergleichender Blick auf die Tabelle in HoppeSeyler-Thierfelders Handbuch der chemischen Analyse, S. 519, wo die Analysen der bisher untersuchten Keratine angeführt sind, und auf die vorstehende Übersicht lehrt uns, daß sich das Schildpattkeratin von den anderen vor allem durch seinen hohen Glykokollgehalt und das Fehlen der Glutaminsäure beträchtlich unterscheidet. Weiters läßt auch die erheblich größere Tyrosinmenge, wodurch sich dieses Material zur Darstellung gerade dieser Aminosäure ganz vortrefflich eignet, einen deutlichen Unterschied erkennen. Endlich ist auch der geringere Gehalt an Leucin auffallend. Fortgesetzte und bereits in Arbeit stehende Untersuchungen verschiedener andersartiger Keratine sollen unsere Kenntnis über den Aufbau dieser Art von Eiweißkörpern noch erweitern.

Graz, 26. Juli 1911. 